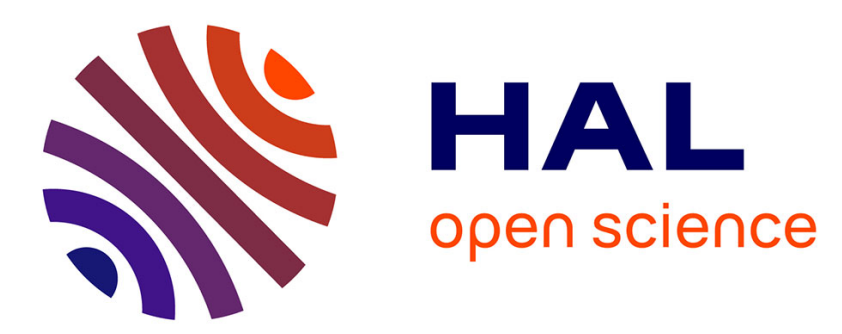

\title{
Intercell interference mitigation for uplink OFDM code division multiplexing
}

Laurent Cariou, Jean-François Hélard, Jean-Yves Baudais

\section{To cite this version:}

Laurent Cariou, Jean-François Hélard, Jean-Yves Baudais. Intercell interference mitigation for uplink OFDM code division multiplexing. IEEE ISSSTA, Aug 2006, Manaus-Amazomn, Brazil. pp.153-157, 10.1109/ISSSTA.2006.311753 . hal-00283663

\section{HAL Id: hal-00283663 https://hal.science/hal-00283663}

Submitted on 18 Sep 2014

HAL is a multi-disciplinary open access archive for the deposit and dissemination of scientific research documents, whether they are published or not. The documents may come from teaching and research institutions in France or abroad, or from public or private research centers.
L'archive ouverte pluridisciplinaire HAL, est destinée au dépôt et à la diffusion de documents scientifiques de niveau recherche, publiés ou non, émanant des établissements d'enseignement et de recherche français ou étrangers, des laboratoires publics ou privés. 


\title{
INTERCELL INTERFERENCE MITIGATION FOR UPLINK OFDM CODE DIVISION MULTIPLEXING
}

\author{
Laurent Cariou, Jean-François Hélard and Jean-Yves Baudais \\ Electronics and Telecommunications Institute of Rennes (IETR) \\ INSA, 20, avenue des buttes de Coesmes, 35043 Rennes, France \\ laurent.cariou@ens.insa-rennes.fr, \{jean-francois.helard,jean-yves.baudais\}@insa-rennes.fr
}

\begin{abstract}
In this paper, we investigate the robustness of the spread spectrum multi-carrier multiple access (SS-MC-MA) technique in uplink multicellular systems. In a first step, a statistical characterization of the intercell interference is carried on to model it for BER performance simulations. Then, the impact of the intercell interference on such a scheme is evaluated and the robustness of SS-MCMA system by mitigating those interferences thanks to frequency allocation, channel coding and spreading gain is optimized.
\end{abstract}

\section{INTRODUCTION}

Nowadays, some of the most promising technologies for the air interface of the fourth generation (4G) terrestrial systems, concerned by flexibility and very high spectrum efficiency, are multi-carrier spread-spectrum techniques (MC-SS). In downlink, multi-carrier code division multiple access (MC-CDMA) combines the merits of orthogonal frequency division multiplex (OFDM) with those of CDMA [1] by spreading users' signals in the frequency domain, offering a strong robustness to multipath propagation. However, in uplink, to reduce channel estimation complexity, spread spectrum multi-carrier multiple access (SS-MC-MA) has been proposed in [2]. SS-MC-MA assigns each user exclusively its own subset of subcarriers according to an additive frequency division multiple access (FDMA) scheme. Thanks to that, the base station (BS) only has to estimate, for each subcarrier, one channel compared to $N_{u}$ for uplink MC-CDMA. Moreover, self interference (SI) can easily be cancelled by single user detection (SD).

The reduction, for each user, of the number of subcarriers with SS-MC-MA, compared to MC-CDMA, can lead to a reduction in the exploitation of the frequency diversity. In order to compensate for this, one can introduce a frequency multiplexing of the users on the whole bandwidth. As an alternative, we recently proposed in [3] to use subsets of adjacent subcarriers and to allocate them to the different users by applying a frequency hopping pattern (FH). In that case, it has been demonstrated that each user benefits as well from the frequency diversity linked to the total bandwidth, while offering a strong robustness to user's oscillators frequency shifts [4]. Combined with Alamouti space-time block code (STBC) [5], the efficiency of the novel scheme STBC FH SS-MC-MA as a promising system for the uplink of the future wideband wireless networks has been successfully demonstrated in the case of perfect and non-perfect channel estimation [6]. However, a lot of work has still to be done concerning multicellular aspects.

When considering the use of such techniques in cellular environments, severe performance degradations due to intercell interferences (ICI) have to be expected and evaluated. This interference level is particularly high when the system is operating with a frequency reuse factor of 1 , meaning that all the BSs transmit in the same frequency band and interfere with each other.

The modelling of the ICI is the first step to get over when looking into cellular aspects. With MC-CDMA systems, it has been demonstrated in [7] that this interference could be considered as gaussian and modelled by an additive white gaussian noise (AWGN) assuming the use of scrambling codes to decorrelate the signals from neighboring cells. This article first focuses on the statistical characterization of the ICI with SS-MC-MA systems and on a way to model it for bit error rate (BER) performance calculations. Then, the impact of the ICI on such a scheme is evaluated and the robustness of SS-MC-MA system by mitigating those interferences thanks to frequency allocation, channel coding and spreading gain is optimized, assuming perfect synchronization between cells and no communications between BSs.

\section{SS-MC-MA SCHEME DESCRIPTION}

Figure 1 shows a simplified SS-MC-MA system for user $j$. As already mentioned in the introduction, SS-MC-MA adds an FDMA scheme at subcarrier level in which each user $j=1, \ldots, N_{u}$ exclusively transmits on a subset of $L$ subcarriers out of a total of $N_{c}=N_{u}$. L. After symbol mapping, a CDMA Walsh-Hadamard spreading process 

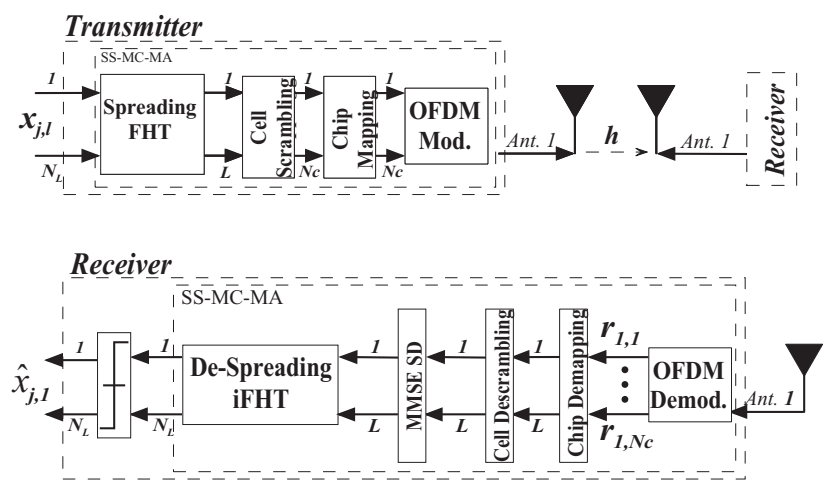

Fig. 1. SS-MC-MA transmitter and receiver for user $j$.

is applied to a defined number $N_{L}$ of selected complexvalued data symbols $x_{l}$, which is equal to the length $L$ of the spreading codes in the full-load case. Those symbols $x_{l}$ can be denoted by $x_{j, l}$ as they all belong to user $j$ with SS-MC-MA.

Those $N_{L}$ selected data symbols are multiplied by their specific orthogonal Walsh-Hadamard spreading code $\mathbf{c}_{l}=\left[c_{l, 1} \ldots c_{l, k} \ldots c_{l, L}\right]^{T}$ and superposed with each other. $c_{l, k}$ is the $k^{t h}$ chip and [.] $]^{T}$ denotes vector or matrix transposition. $\mathbf{c}_{l}$ is the $l^{\text {th }}$ column vector of the $L \times N_{L}$ spreading code matrix $\mathbf{C}$. The resulting CDMA spread symbol $\mathbf{s}^{(j)}=\left[s_{1}^{(j)} \ldots s_{k}^{(j)} \ldots s_{L}^{(j)}\right]$ can be expressed as follows:

$$
s_{k}^{(j)}=\sum_{l=0}^{N_{L}-1} x_{j, l} c_{l, k} \quad k=1, \ldots, L
$$

The chips $s_{k}^{(j)}$ of a CDMA spread symbol are then transmitted in parallel over a subset of $L$ subcarriers among $N_{c}$ proposed by the OFDM modulation. Hence, assuming that $N_{c}$ is a multiple of $L$, the system can support $N_{u}$ spread symbols $s^{(j)}$, each user possessing its own spread symbol and its own subset of subcarriers. The chip mapping component determines this subset. This subcarriers selection, called frequency allocation is more deeply presented in section V.

At the reception, the OFDM demodulation is carried out by a simple and unique fast Fourier transform (FFT) applied to the sum of the $N_{u}$ different users' signals. After de-spreading and threshold comparison, the detected data symbols $\hat{x}_{j, l}$ for user $j$ are:

$$
\hat{x}_{j, l}=\mathbf{c}_{l}^{T} \mathcal{G} \mathcal{Y}=\mathbf{c}_{l}^{T} \mathcal{G H} \mathcal{H}
$$

where $\mathcal{Y}=\left[y_{1} \ldots y_{k} \ldots y_{L}\right]$ is the vector of the received signals, $G$ and $H$ are the diagonal matrices containing the equalization coefficients for the propagation channel and the channel coefficients respectively. In order to detect the $N_{L}$ transmitted symbols $x_{j, l}$, for the desired user $j$, minimum mean square error (MMSE) single user detection (SD) scheme is applied to the received signals.
Furthermore, UMTS turbo-coding associated with time interleaving, not represented in this figure, are considered.

\section{MULTI-CELLULAR HYPOTHESIS}

When tackling with cellular aspects of wireless communication, a few assumptions have to be made concerning synchronization, communication between BSs and interference modelling.

Perfect synchronization between cells : In our work, we have considered a system operating with a frequency reuse factor of 1, meaning that all the BSs transmit in the same frequency band. Furthermore, synchronization between cells is assumed, which means that all the BSs follow the same uplink frame structure. With SS-MC-MA scheme, when the delay between the different received signals is lower than the guard interval duration, the orthogonality between the subcarriers is kept in the receiver, if the drifts between the oscillators frequencies are negligible.

Assuming a system with automatic gain control (AGC), the power transmission of mobile terminals (MT) far from their BS is bigger than the one of MTs close to it. Thanks to that, only the MTs close to the border between two cells will have a significant intercell interfering impact. As those MTs are approximately localized at equal distance from their BSs and from the neighboring one, the delay between the interfering and useful SS-MC-MA signals will be lower than the guard interval duration and the orthogonality between subcarriers will be preserved.

No communication between BSs : We consider that no information is available concerning the interfering signals and that the system must be able to operate at full capacity. This article will then focus on the mitigation of the cellular interferences thanks to frequency allocation, spreading gain and channel coding under these assumptions.

\section{NATURE OF THE INTERCELL INTERFERENCE WITH SS-MC-MA}

For each user $j$, the SS-MC-MA signal observed before the OFDM modulation at the transmission side is a CDMA signal made up of chips $s_{k, n}^{j}$, that corresponds to the data transmitted on a subcarrier $k$ and during OFDM symbol $n$. Constituted of a sum of $N_{L}$ discrete uniform random variables, it can be considered as gaussian with zero mean and a variance equal to the transmitted power $P$, thanks to the central limit theorem as $N_{L}$ grows. Moreover, cell scrambling codes csc $_{m}=\left[c s c_{m, 1} \ldots c s c_{m, k+L * n} \ldots c s c_{m, L * N_{f}}\right]^{T}$ are used at a frame level on each cell $m$ to decorrelate the signals from different cells. $N_{F}$ is the number of SS-MC-MA symbols per frame. Therefore, each chip can be considered as white gaussian and expressed by:

$$
s_{k, n}^{j}=\left(\sum_{l=0}^{N_{L}-1} x_{l}^{j} \cdot c_{l, k}\right) \cdot \csc _{(m, k+L * n)}=\mathcal{N}(0, P)
$$




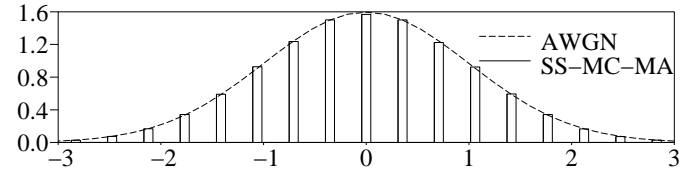

Fig. 2. SS-MC-MA probability density function (pdf) at the transmitter

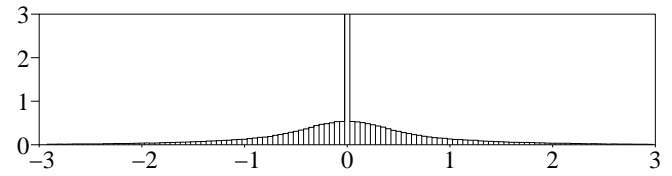

Fig. 3. Intercell interference probability density function (pdf)

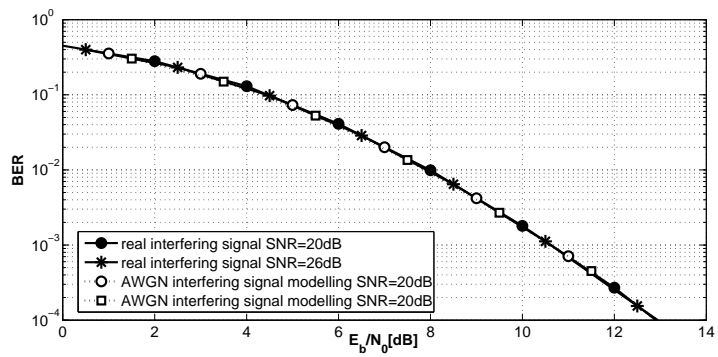

Fig. 4. Performance comparison with a real version and an AWGN modelling of the interference signal; full load coded SS-MC-MA systems; Rayleigh SISO model; MMSE SD, QPSK, channel coding rate $\mathrm{R}=1 / 2$.

The probability density function (pdf) of the real part of SS-MC-MA signal is presented on figure 2 and compared to the one of the normal distribution with an equal variance.

If we now consider the received SS-MC-MA signal observed after the OFDM demodulation at the reception, i.e. after FFT process, it corresponds to the chip of a CDMA signal weighted by a cell scrambling pattern multiplied by the frequency response of the channel between the interfering MT and the observed BS. Under cellular synchronization assumption, on each subcarrier, the interfering signal $I_{k, f}^{j}$ from one user $\mathrm{j}$ is thus in the form of a white gaussian term weighted by a frequency channel coefficient.

$$
\begin{gathered}
I_{k, n}^{j}=s_{k, n}^{j} \cdot h_{k, n}^{j}=\left(\sum_{l=0}^{N_{L}-1} x_{l}^{j} \cdot c_{l, k}\right) \cdot \csc _{(m, k+L * n)} \cdot h_{k, n}^{j} \\
I_{k, n}^{j}=\mathcal{N}(0, P) \cdot h_{k, n}^{j}
\end{gathered}
$$

The pdf of the real part of this signal is presented on figure 3 in the case of a Rayleigh channel. It approaches the distribution of the multiplication of two discrete gaussian terms. Figure 4 gives the BER performance of a full-load SSMC-MA system over a Rayleigh channel with two types of ICIs, either the scrambled full-load SS-MC-MA signal (true version) or the AWGN signal (modelling version). The simulations are run with convolutional channel coding of rate $1 / 2$ and MMSE SD. The results are given in terms of bit energy to noise plus interference ratio $\left(E_{b} /\left(N_{0}+I\right)\right)$ and with two fixed $E_{b} / N_{0}$ values of 20 and $26 \mathrm{~dB}$.
Although the modelling and true versions of the ICI don't share identical statistical characteristics (the former is a multiplication of two white gaussian terms, the latter a simple white gaussian term), their impact on BER performance are the same. This can be seen by two ways. First, with fixed $E_{b} / N_{0}$ values, the curves with true and modelling versions juxtapose themselves. Secondly, with fixed $E_{b} /\left(N_{0}+I\right)$ values, the results of the true version with $E_{b} / N_{0}$ equal to 20 and $26 \mathrm{~dB}$ are the same, underlying the fact that AWGN noise and intercell interference have the same impact. We can therefore assert that for BER performance comparison, one can model the SS-MC-MA ICI with a simple AWGN term.

\section{FREQUENCY ALLOCATION WITH SS-MC-MA}

\section{V-A. Mono-cellular criteria}

The frequency allocation determines the subset of subcarriers on which the spread symbols' chips from user $j$ are distributed. In mono-cellular systems, the optimization criteria of the frequency allocation are the frequency diversity exploitation, the resulting channel estimation complexity, the robustness against the frequency drifts between the different terminals oscillators.

Block Interleaved SS-MC-MA (BI) : Frequency multiplexing can be performed to achieve block interleaved SSMC-MA, denoted $B I$ in figure 5. Basically, it distributes the chips of a spread symbol on the whole bandwidth in order to take benefit of the full frequency diversity.

SS-MC-MA with adjacent subcarriers subsets (AS) : This allocation simply distributes the chips of a spread symbol on a limited bandwidth by using subsets of adjacent subcarriers. Those two solutions are sub-optimal. While the former leads to an increase of the channel estimation complexity and a weak robustness against oscillators frequency drifts [4], the latter only benefits from the frequency diversity linked to a limited bandwidth.

SS-MC-MA with AS and frequency hopping (AS FH) As an alternative, we recently proposed in [3] to use subsets of adjacent subcarriers and to allocate them to the different users by applying a frequency hopping pattern $(\mathrm{FH})$. The FH pattern respects a simple law $b_{i, j}=$ $\left(b_{i-1, j}+i n c\right) \bmod N_{s}$ which allows each user to experiment equiprobably each frequency subset, while avoiding collisions between users. $s_{i, j}$ is the subset index for OFDM symbol $i$ and user $j, N_{s}$ is the number of subsets per OFDM symbol and inc the subset index increment selected to optimally take advantage of the frequency diversity.

In that case, it has been demonstrated that each user benefits from the frequency diversity linked to the total bandwidth, while offering a strong robustness to user's oscillators frequency shifts [4]. In [6], a low channel estimation complexity has been proposed for this scheme. 


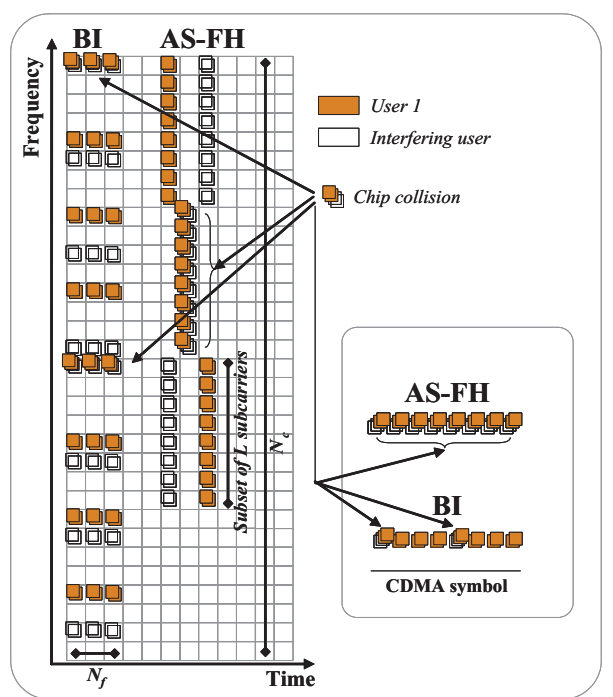

Fig. 5. Frequency allocation solutions plotted on the OFDM twodimensional frame.

\section{V-B. Multi-cellular criteria}

In multi-cellular systems, besides the previous criteria, we also have to weigh up the interference averaging between users for fairness considerations and the efficiency of the spreading gain offered by the CDMA dimension in order to mitigate the ICI.

With $A S F H$ technique, the use of different $\mathrm{FH}$ patterns between cells averages perfectly the interferences among users but doesn't offer any spreading gain benefits. When a collision occurs, i.e. when two users from neighboring cells transmit on the same subset of subcarriers, all the chips of the interfered CDMA symbol are corrupted identically. In the worth case, the interfering power can be equal to the useful signal power. Assuming $p$ the collision probability, the BER performance results can then be expressed by :

$$
\begin{gathered}
B E R=(1-p) \times B E R_{E_{b} / N_{0}}+p \times B E R_{E_{b} /\left(N_{0}+I\right)} \\
B E R \simeq p \times B E R_{E_{b} /\left(N_{0}+I\right)}
\end{gathered}
$$

With the $B I$ technique, on the other hand, the use of different interleaving patterns between cells averages perfectly the interferences among users and permits to benefit from a spreading gain. The collisions occur on given subcarriers during the whole frame, as represented in figure 5, with a collision probability $p$. Then, only a few chips of the CDMA symbols are corrupted. At the despreading process, those interferences are averaged over the whole CDMA symbol exhibiting a spreading gain. The BER performance results can be expressed by :

$$
B E R=B E R_{E_{b} /\left(N_{0}+p \times I\right)}
$$

SS-MC-MA with AS, FH and interleaved spreading (AS FH ISp) : In order for the $A S F H$ solution to take advantage of the spreading gain and then, to benefit from the advantages of both schemes, we propose to add to the $A S F H$ solution a chip interleaving before the chip mapping. Assuming that $N_{f}$ is a multiple of $L$, the subset $n$ of subcarriers, i.e. transmitted on OFDM symbol $n$, is filled with the $n^{\text {th }}$ chips of the $L$ CDMA symbols instead of the $L$ chips of the $n^{t h}$ CDMA symbol as with $A S F H$.

The collisions occur subset by subset as with $A S F H$ but, due to the chip interleaving, only a few chips of the CDMA symbols are corrupted as with $B I$. Thus, it can benefit from the same spreading gain as with $B I$ while keeping the advantages of the $A S F H$ technique and fulfill all the mono and multi-cellular criteria.

\section{SIMULATION RESULTS}

\section{VI-A. System parameters}

The system parameters are chosen according to the time and frequency coherence of the channel in order to match the requirements of a $4 \mathrm{G}$ mobile cellular system. The carrier and sampling frequencies are set to $5 \mathrm{GHz}$ and $57.6 \mathrm{MHz}$ respectively. The FFT size equals 1024 and the number of modulated subcarriers reaches 736 . The guard interval duration is equal to $3.75 \mu \mathrm{s}$ and the total symbol duration $21.52 \mu s$. Channel coding is composed of a rate $1 / 3$ UMTS turbo-coding followed by puncturing pattern defined to achieve a global coding rate $R$ of $1 / 2$. The time interleaving depth has been adjusted to the frame duration made of $N_{f}=32$ OFDM symbols. With a QPSK modulation, this leads to an asymptotical spectrum efficiency of $1 \mathrm{bit} / \mathrm{s} / \mathrm{Hz}$. We use a link level SISO channel model which uses the BRAN E channel average power delay profile (APDP), refering to a typical outdoor urban multi-path propagation. The measured coherence bandwidth monosided at $3 \mathrm{~dB}$ is roughly $1.5 \mathrm{MHz}$. Finally, when the MT velocity is $72 \mathrm{~km} / \mathrm{h}$, the time correlation remains close to the frame duration.

\section{VI-B. Numerical results}

All figures show the BER performance results of the scrambled SS-MC-MA system in the presence of AWGN ICI modelled by one user in a neighboring cell, presented in terms of bit energy to interference ratio $\left(E_{b} / I\right)$ for a given $E_{b} / N_{0}$.

The comparison between the two optimum frequency allocation, $A S F H$ and $A S F H$ with interleaved spreading $I S p$ is presented in figure 6 without channel coding to highlight the spreading gain benefits. Perfect channel estimation is considered, the probability of collision $p$ is fixed to $1 / 32$ and $1 / 8$ respectively. In order for the two solutions to share the same diversity order, the results are presented over the AWGN channel with a $E_{b} / N_{0}$ fixed to $8 \mathrm{~dB}$. Then, in that first step, there are no intracell interferences called self interferences (SI) with SS-MCMA. In both cases, the curves behavior is shown to be 


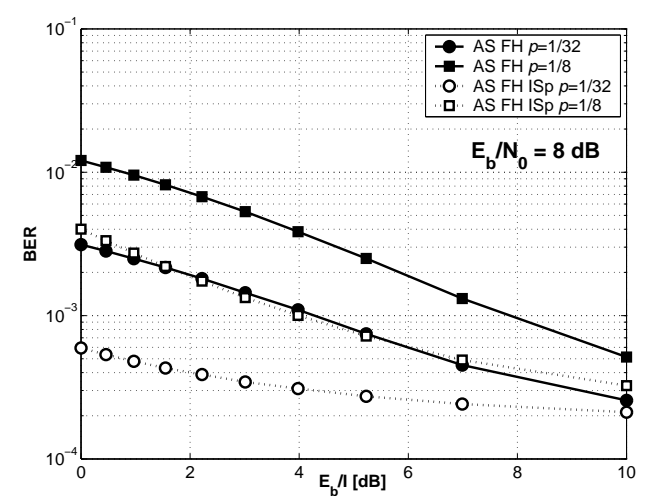

Fig. 6. Comparison of different frequency allocation techniques without channel coding; AWGN SISO model; full load SS-MC-MA; perfect channel estimation, MMSE SD, QPSK, $E_{b} / N_{0}=8 d B$.

suitable with the theoretical calculations. It demonstrates that $A S F H I S p$ solution is significantly more robust than $A S F H$ against the ICI without channel coding thanks to the spreading gain.

Figure 7 shows the performance results over a BRAN E SISO channel with $E_{b} / N_{0}$ fixed to $12 \mathrm{~dB}$, with channel turbo coding and the embedded pilot channel estimation presented in [6] taking into account the pilot insertion power losses and a collision probability of $p=1 / 8$. The two solutions still share the same diversity order thanks to the channel coding [3] but the SI is now non-negligible due to the selectivity of the channel. Two different cases are evaluated. The first one considers that the $E b / I$ is not known at the reception while the second assumes this knowledge, meaning that the collision localization has been estimated previously.

At $E_{b} / I=10 \mathrm{~dB}$, the ICI term is negligible. As expected, $A S F H$ solution exhibits better results than $A S F H I S p$ because the SI is lower with $A S F H$ than with $A S F H$ ISp. In fact, it has been demonstrated in [6] that the SI is directly linked to the variance of the channel on which the chips of the spread symbol are distributed. This variance is lower with $A S F H$ than with $A S F H I S p$.

Without knowing the interference power level, neither the MMSE detection nor the channel decoding are optimum. The curve behavior underline the interest of the spreading gain especially for low interference power level. We can therefore affirm in that case, that $A S F H I S p$ is more robust against ICI but more sensitive to intra-cell interferences. As the intra-cell interferences are known within the cell, it can be more easily cancelled with a little increase of complexity.

Knowing the interference power level and inserting it in the confidence value calculation, MMSE detection and channel decoding can be optimized. Channel decoding is more efficient with $A S F H$ as the interferences are localized instead of being spread on all the transmitted data like with $A S F H I S p$. In that case, $A S F H$ appears to be more robust

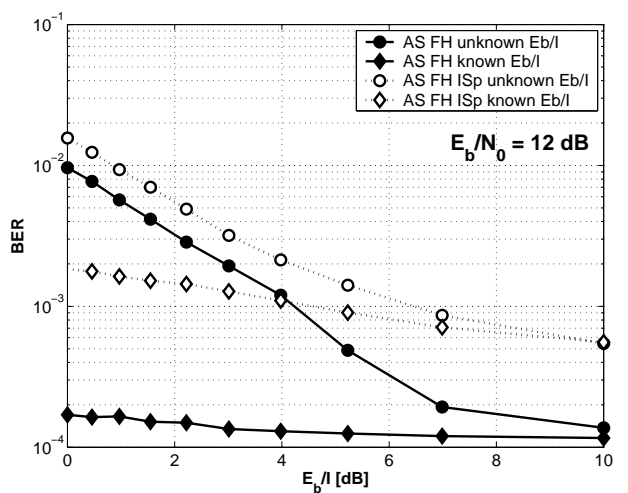

Fig. 7. Comparison of different frequency allocation techniques with channel coding; BRAN E SISO model; full load turbo-coded SS-MC-MA; embedded pilot channel estimation, MMSE SD, QPSK, $R=1 / 2, p=1 / 8$, MT velocity of $72 \mathrm{~km} / \mathrm{h}, E_{b} / N_{0}=12 d B$.

against intra and intercell interferences without benefiting from the spreading gain.

\section{CONCLUSION}

In this article, we have first shown that the intercell interference with uplink SS-MC-MA systems can be modelled by an AWGN signal for BER performance simulations. Then, the robustness of SS-MC-MA systems has been evaluated by comparing different interference mitigation solutions. It has been demonstrated that without channel coding, AS FH ISp solution is more robust than ASFH against intercell interferences. On the contrary, with channel coding and optimized confidence value calculation, $A S$ $\mathrm{FH}$ is more robust on frequency selective channels against intra and intercell interferences.

\section{ACKNOWLEDGMENTS}

The authors would like to thank France Telecom R\&D/RESA/BWA which supported this work within the contract 46128187.

\section{REFERENCES}

[1] S. Hara and R. Prasad, "Overview of multicarrier CDMA," IEEE communication magazine, vol. 35, no. 12, pp. 126-133, Dec. 1997.

[2] S. Kaiser and W.A. Krzymien, "Performance effects of the uplink asynchronism in a spread spectrum multi-carrier multiple access system," Europeen Transactions on Telecomunications, vol. 10, no. 4, pp. 399-406, July/August 1999.

[3] L. Cariou and J.F. Helard, "MIMO Frequency Hopping Spread Spectrum Multi-Carrier Multiple Access: A Novel Uplink System for B3G Cellular Networks," Telecommunication Systems, vol. 30, no. 1-3, pp. 193-214, Nov. 2005.

[4] A. Arkhipov and M. Schnell, "The influence of user frequency offset on the uplink performance of SS-MC-MA," Proc. of European Conference on Wireless Technology (ECWT'03), pp. 451-454, Oct. 2003.

[5] S.M. Alamouti, "A simple transmit diversity technique for wireless communication," IEEE Journal on Selected Areas in Communications, vol. 16, pp. 1451-1458, Oct. 1998.

[6] L. Cariou and J.F. Helard, "Superimposed pilot-based channel estimation for MIMO OFDM Code Division Multiplexing uplink systems," in Fifth International Workshop on Multi-Carrier Spread Spectrum (MCSS'05), Oberpfaffenhofen, Germany, Sept 2005.

[7] X.G. Doukopoulos and R. Legouable, "Impact of the intercell interference in DL MC-CDMA systems," in Fifth International Workshop on Multi-Carrier Spread Spectrum (MCSS'05), Oberpfaffenhofen, Germany, Sept 2005. 